\title{
OPEN Evaluation on reprogramed biological processes in transgenic maize varieties using transcriptomics and metabolomics
}

\author{
Wei Fu' ${ }^{1}$, Pengyu $\mathrm{Zhu}^{1}$, Mingnan $\mathrm{Qu}^{2}$, Wang $\mathrm{Zhi}^{1}$, Yongjiang Zhang ${ }^{1}$, Feiwu $\mathrm{Li}^{3 \otimes} \&$ \\ Shuifang Zhu $\mathbf{H}^{1 \bowtie}$
}

Genetic engineering (GM) has great potential to improve maize productivity, but rises some concerns on unintended effects, and equivalent as their comparators. There are some limitations through targeted analysis to detect the UE in genetically modified organisms in many previous studies. We here reported a case-study on the effects of introducing herbicides and insect resistance $(H I R)$ gene cassette on molecular profiling (transcripts and metabolites) in a popular maize variety Zhengdan958 (ZD958) in China. We found that introducing HIR gene cassette bring a limited numbers of differential abundant genes (DAGs) or differential abundant metabolites (DAMs) between transgenic events and non-transgenic control. In contrast, averaged 10 times more DAGs and DAMs were observed when performed comparison under different growing environments in three different ecological regions of China than the numbers induced by gene effects. Major biological pathways relating to stress response or signaling transduction could explain somehow the effects of growing environments. We further compared two transgenic events mediated ZD958 (GM-ZD958) with either transgenic parent GM-Z58, and other genetic background nonGM-Z58, nonGM-ZD958, and Chang7-2. We found that the numbers of DAGs and DAMs between GM-ZD958 and its one parent maize variety, Z58 or GM-Z58 is equivalent, but not Chang7-2. These findings suggest that greater effects due to different genetic background on altered molecular profiling than gene modification itself. This study provides a case evidence indicating marginal effects of gene pleiotropic effects, and environmental effects should be emphasized.

Genetic engineering plays crucial roles in crop productivity improvement; however, it may possess sometimes unintended effects on the crop quality and the public health. It has been extensively applied in the improved resistance to disease, herbicides as well as crops quality, especially in maize, as the most widely grown crop worldwide, and contributes to around 800 million tons production from 2007 to $2008^{1}$. Budget allocations for major biotechnology funding agencies has been annually increased in several countries. The Indian government allocates an estimated US\$15 million annually on plant biotechnology sector, while the private sector contributes about US $\$ 10$ million $^{2}$. China currently accelerates its investments in agricultural biotechnology research and is focuses further on the commodities that have been mostly ignored in the laboratories of industrialized countries ${ }^{2}$.

However, the derived products from genetically modified crops (GM) always rises controversy, especially in Europe, despite the absence of evidences proving risks of GM crops. The major concern from public community is about whether GM crops are subjected to critical inspection regarding food safety ${ }^{3}$. It has been agreed internationally to ensure a promising equivalence between GM crops and traditional varieties ${ }^{4}$. The biological effects of GM crops on human health, environment, agronomy, and economy were extensively reported, and some researchers found that no obviously adverse-effects attributed to genetic engineering on the human population ${ }^{5}$. Another concern about the unintended effects on the alteration of DNA sequences, proteins or new metabolites or reprogramed metabolites due to random insertion on genomic regions ${ }^{6,7}$, created by Agrobacterium-mediated gene transfer and particle bombardment ${ }^{8-10}$. Therefore, it needs to be deeply investigated via a specific powerful

${ }^{1}$ Institute of Plant Quarantine, Chinese Academy of Inspection and Quarantine, Beijing 100176, China. ${ }^{2}$ National Key Laboratory of Plant Molecular Genetics, CAS Center for Excellence in Molecular Plant Sciences, Shanghai 200032, China. ${ }^{3}$ Institute of Agricultural Quality Standard and Testing Technology, Jilin Academy of Agricultural Sciences, Changchun 130033, Jilin, China. ${ }^{\boxplus}$ email: lifeiwu3394@sina.com; zhushf0421@163.com 
A

Generation of ZD958 transgenic lines

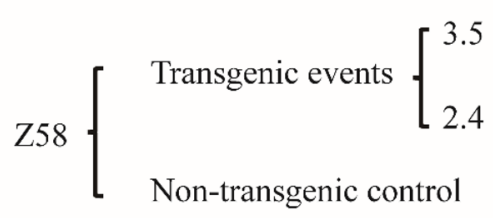

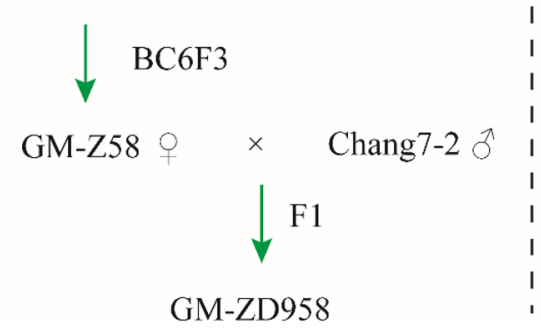

B

Generation of ZD958

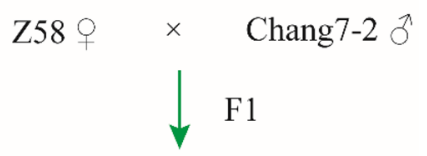

nonGM-ZD958

Figure 1. Pipeline of transgenic maize lines in this study. (A) The transgenic Zhengdan958 (GM-ZD958) was produced by agrobacteria-mediated transformation into Zheng58 (Z58) and then was used to hybrid with Chang7-2. (B) Non-transgenic ZD958 (non-GM ZD958) was used as control produced from Z58 and Chang72.

\begin{tabular}{|l|l|l|l|l|l|l|}
\hline Type & Material & $\begin{array}{l}\text { Transgenic event } \\
\mathbf{3 . 5}\end{array}$ & $\begin{array}{l}\text { Transgenic event } \\
\mathbf{2 . 4}\end{array}$ & Non-GM control & Growth chamber & Field experiments \\
\hline Inbred line & Zheng 58 & $\sqrt{ }$ & $\sqrt{ }$ & $\sqrt{ }$ & $\sqrt{ }$ & - \\
\hline Inbred line & Chang 7-2 & - & - & $\sqrt{ }$ & $\sqrt{ }$ & - \\
\hline Hybrid variety & Zhengdan 958 & $\sqrt{ }$ & $\sqrt{ }$ & $\sqrt{ }$ & $\sqrt{ }$ & $\begin{array}{l}\text { Beijing, Zheng- } \\
\text { zhou, Harbin }\end{array}$ \\
\hline
\end{tabular}

Table 1. Transgenic and non-transgenic maize lines used in this study.

and comprehensive analyzing method to compare the GM crops or products to their counterparts from parental or near isogenic lines ${ }^{11,12}$.

Recently, the roles of emerging "omics" technologies in the assessment of unintended gene effects was extensively proposed, the omic analysis has been successfully applied for the reconstruction of genome-scale networks in some commercialized GM crops $^{13-15}$. However, most studies performed the targeted analysis, which may, have its limitations in detecting unintended effects in genetically modified organisms ${ }^{12}$. Consequently, this prompted us to further assess the non-targeted profiling or fingerprinting technologies that could be used as unbiased analytical approaches to characterize the potential occurrence of the unintended effects caused by introducing exogenous gene or overexpressing endogenous gene ${ }^{13}$.

The objective of this investigation is to gain more insights and perform a cutting edge research using high technologies and targeted omics analysis to a deeply understand of the biology of the Zhengdan958 GM maize by molecular profiling (transcriptomes and metabolomics). Herein, we investigated molecular profiling to provide evidences into the extent of variation in the maize transcriptome and metabolome by analyzing a multiple gene cassette underlying both herbicide and insect resistance (named HIR cassette) engineered into different maize backgrounds. These included two transgenic modified lines and the respective control line. All are commercial lines and were grown in different locations in china. We report on the application of transcriptome profiling, and capillary liquid chromatographic/mass spectrometric (LC/MS)-based metabolite profiling for the analysis of metabolome. We performed GO and KEGG analyses to identify the potentially altered biological processes and/ or metabolic pathways that were mainly responsible for the differences between GM lines and their respective non-GM maize lines. The data presented serve as an exploratory study into the use of 'omics' approaches for a safety and successfully evaluation of GM crops.

\section{Results}

Generation of HIR cassette transgenic maize lines. Herbicide and insect resistant are two major concerns in maize breeding. In this study, we transformed a HIR gene cassette consisting of EPSPS and Cry $1 A b$ gene underlying herbicide and insect, respectively, into maize cultivar Zheng58 (Z58), forming a gene modified (GM) line (GM-Z58). Then we created a maize line in BC6F3 generation, GM-ZD958, through crossing GM-Z58 and hybrid non-GM maize variety Chang7-2. In this regard, we performed two independent transgenic events, i.e., event-2.4 and event-3.5 using same vectors harboring same gene, to elucidate the random insertion effects of targeted genomic regions (Fig. 1, Table 1). 
A

B

Herbicide and insect resistence (HIR) cassette

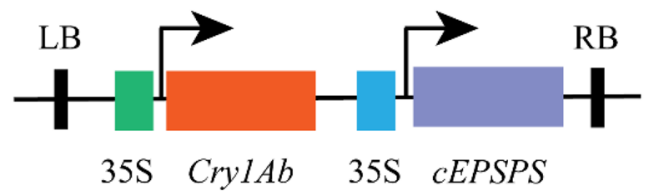

D

Early stage insect infection

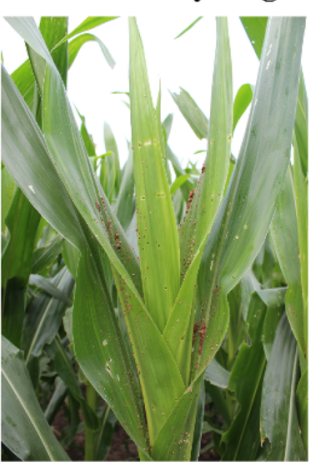

ZD958

$\mathbf{F}$

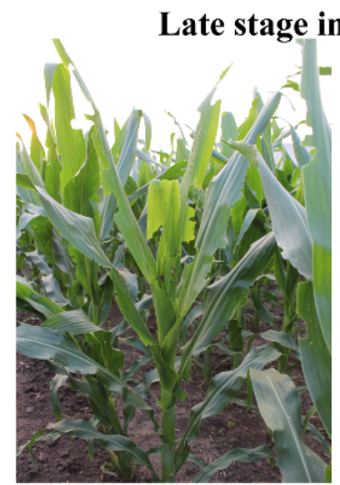

ZD958

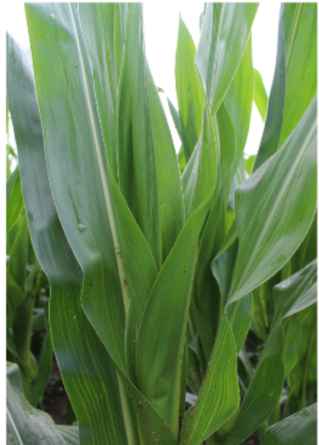

GM-ZD958

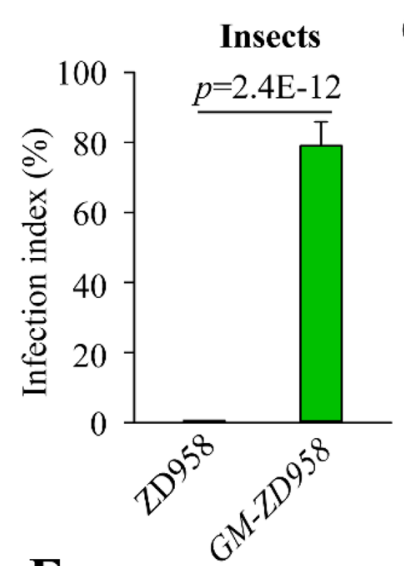

C

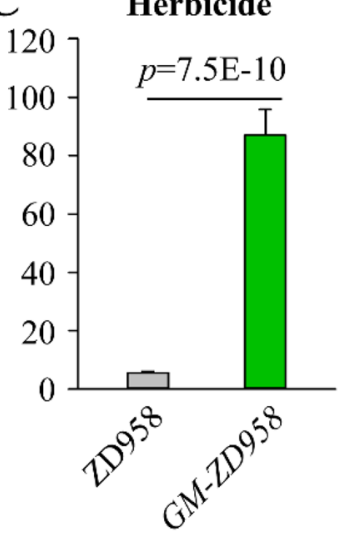

$\mathbf{E}$

Insects infection

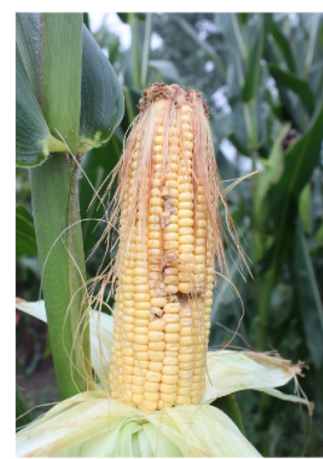

ZD958

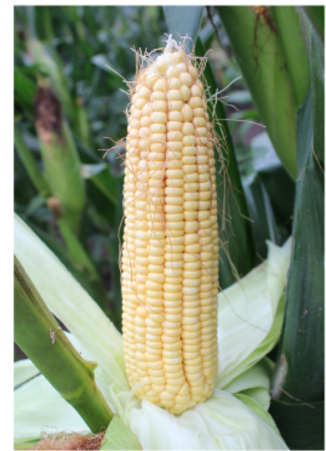

GM-ZD958

G

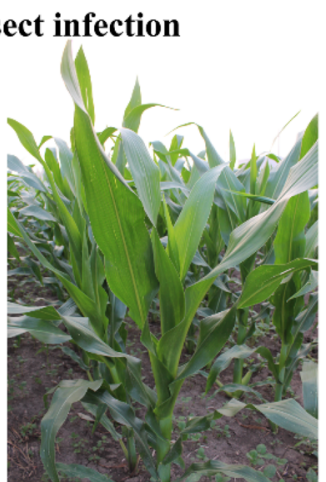

GM-ZD958

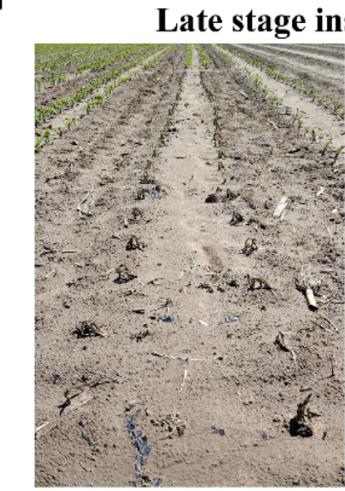

ZD958

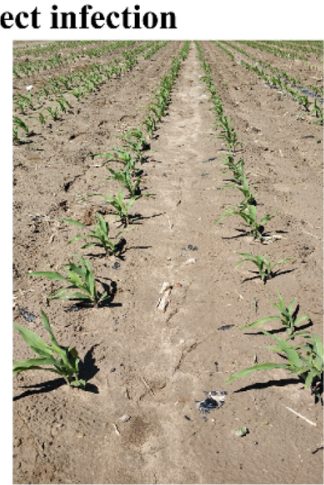

GM-ZD958

Figure 2. Field performance of herbicides and insect resistance of ZD958 expressing HIR gene cassette in genemodification (GM)-ZD958 maize lines grown in Beijing field (E116.3 $3^{\circ}, \mathrm{N} 39.7^{\circ}$ ), China. (A), Schematic map of constructed vector for HIR gene cassette. (B,C), Comparison of infection index to insect (Bacillus thuringiensis) and herbicide (glyphosate) resistance in GM-ZD958 from two transgenic events, compared to non-GM ZD958. (D,E), Field imaging of GM-ZD958 and non-GM ZD958 exposed naturally to insect in canopy in early stage (D) and maize seeds during silking stage (E). (F,G), Field imaging of GM-ZD958 and non-GM ZD958 exposed naturally to insect in canopy in late stage (F) and performance of non-GM ZD958 and GM-ZD958 subjected to glyphosate treatments.

Transgenic vector harboring HIR gene cassette fusion with $35 \mathrm{~S}$ promoter were constructed (Fig. 2A). The derived maize variety, GM-ZD958 for two transgenic events (event-2.4 and event 3.5) exerted stronger resistance to either herbicide or insect than GM-Z58 and Chang7-2 (Fig. 2B-G). Non-GM ZD958 maize lines were used as control (CK). We first performed a global-mRNA expression analysis on totally 36 datasets of experimental sites, maize genetic background, and transgenic vectors/CK. Our results suggest that there are averaged 47.3 million and 46.2 million for raw and clean reads across 36 datasets, respectively. The account of clean reads over total reads is $97.6 \%$ (Supplementary Table S2). Mapped reads and ratio of mapped against raw reads are 42 million and $91.6 \%$, respectively. Among mapped reads, uniquely mapped reads account for $95.5 \%$. In addition, $96 \%$ mapped 
reads were identified on exon of gene body, while only $1.8 \%$ and $1.9 \%$ were identified in intron and intergenic region of gene body, respectively (Supplementary Table S2).

Different transgenic events possess similar DAGs and DAMs. We have grown the transgenic lines against ZD958 in Beijing using two transgenic events and non-GMZD958 (CK) (Fig. 3). Our results based on principal components analysis (PCA) suggest that two transgenic events show similar clusters of differently abundant genes (DAGs) and differently abundant metabolites (DAMs), while non-GM ZD958 were separated apart from the two transgenic events (Fig. 3A,B). In addition, we found that there are $0.5 \%, 1.1 \%$ and $1.0 \%$ DAGs out of 39,180 total annotated genes in event- 2.4 vs 3.5, event-2.4 vs CK and event-3.5vs CK, respectively (Fig. 3C). Among these DAGs, only 17 genes were uniquely performed in event-2.4 vs 3.5, in which $71 \%$ and $33 \%$ DAGs are either overlapped with event-2.4 vs CK, or event-3.5 vs CK (Fig. 3C, Supplementary Table S3). We also observed that the numbers of overlapping DAGs were more than that unique DAGs occurred in combination of either transgenic events or transgenic event vs CK (Fig. 3C). These results suggest similar effects between two independent transgenic events on DAGs in ZD958 grown in Beijing, indicating, in fact, that greater effect between two transgenic events and CK was observed than that effect between two transgenic events each other.

Consisted with results of transcriptomes, those of metabolic reprogramming analysis show only 34 DAMs out of 2214 total identified metabolites in event-2.4 vs 3.5, while 94 and 97 DAMs were identified in event-2.4 vs CK and event-3.5 vs CK, respectively. In addition, 97\% DAMs in event- 2.4 vs 3.5 were overlapped with either event- 2.4 vs CK or event-3.5 vs CK (Fig. 3D; Supplementary Table S4). These evidences propose that the marginal effects of introducing HIR gene cassette on the unfavorable biological process or metabolic pathways into ZD958.

To uncover the regulation of HIR gene cassette effects on global mRNA expression and metabolites, we compared the transcriptomes and metabolism between event 3.5 and CK. Our results show that among 374 DAGs, 179 and 195 were down- and up-regulated, respectively (Fig. 3E). We also found that 47 and 50 DAMs were down- and up-regulated, respectively (Fig. 3F). Therefore, introducing HIR gene cassette in transgenic event 3.5 leads to even numbers for both down- and up-regulation genes (Fig. 3E). The case is also true for the numbers of DAMs (Fig. 3F).

To further confirm the gene expression levels and metabolite contents regulated by the HIR gene cassette, we tested top 5\% up-regulated genes among 307 DAGs using qPCR and 78 DAMs using targeted metabolites techniques (LC-MS/MS) as listed in Tables 2 and 3. The results confirmed that 17 genes covering different biological pathways were remarkably up-regulated by introducing HIR gene cassette, and its expression levels in two transgenic events (events 3.5 and 2.4) for GM-ZD958 were around $3 \sim 20$ times higher than its non-GM parents, including Chang 7-2 and Z58 (Fig. 4; Supplementary Datasets 1-6). We also found two overlapped DAMs in the two events, including L-Asparagine and Galacturonic acid (Fig. 5; Table 3). Using targeted-metabolic analysis, we quantitatively determined the amount of these two metabolites and confirmed their high abundance in transgenic lines than in their corresponding non-GM parent maize lines.

Experimental effect as a major driving force for both DAGs and DAMs. To test whether different experiments sites have robust effects on DAGs and DAMs, we compared global mRNA expression and the relative abundance of metabolites between GM-ZD958 and non-GM ZD958 (CK) maize lines in three experimental sites of China, i.e., Beijing, Harbin, and Zhengzhou (Fig. 6). Our results show as well that clear separated clusters were observed among the three experimental sites regarding either different transcripts or metabolites, especially between Harbin and Zhengzhou (Fig. 6A,B). We found that 2952 DAGs between the two transgenic events (3.5 and 2.4) and CK in Harbin were not overlapped with either Beijing or Zhengzhou. Similarly, this is true for 505 metabolites measured form Harbin site (Fig. 6C,D; Supplementary Tables S4, S5). The numbers for DAGs induced by environments, for example in Harbin was 8 times higher than that induced solely by the effects of transgenic events compared to CK under growth chamber conditions (Figs. 3C and 6C; Supplementary Table S5), and similar effects were also recorded for DAMs (Figs. 3D and 6D; Supplementary Table S6). Therefore, the above findings suggest that environment has greater effects on the numbers of global molecular profiling than the gene additional effects.

We further found that 1132 and 894 genes were down- and up-regulated in Zhengzhou over Harbin, respectively (Fig. 6E). In the same regards, we recorded 495 and 309 down- and up-regulated metabolites in Harbin over Zhengzhou, respectively (Fig. 6F). In addition, we performed GO and KEGG analysis on the DAGs among three experimental sites based on one-way ANOVA $(p<0.05)$. Results show that biological pathways in the list of catabolic process, organic substance catabolic process, alpha-amino acid metabolic process, intracellular signal transduction and phophorelay signal transduction system were significantly enriched (Fig. 7A). In addition, many metabolic pathways were enriched such as sesquiterpenoid and triterpenoid biosynthesis, arginine and limonene and pinene degradation, brassinosteriod biosynthesis (Fig. 7B). We further picked up top 5\% of DAGs according to $p$-value among three experimental sites based on one-way ANOVA, and the list of these DAGs were shown in Table 2. We found most of genes related to abiotic stress inducible proteins, such as $H t f B-2 b, D n a J$ and $P P R$ gene family, and some transcription factors, such as ZIPs, WEB, DNA-binding-TFs. This suggest that environmental effects induce multiple alteration of biological pathways, and the alteration is above the expectation on herbicide and disease response.

Marginal effects of transgenic HIR gene cassette on biological process. In order to illustrate the effects of transgenic backgrounds on biological gene expression and metabolites, we performed an integrative analysis on transcriptomes and metabolism in GM-Z58 across two transgenic events (event-2.4 and 3.5), nonGM Z58, GM-ZD958 across two transgenic events and non-GM ZD958 (Fig. 8). Results from PCA on relative expression of gene and metabolites, show that the high similarity was observed between transgenic lines harbor- 
A

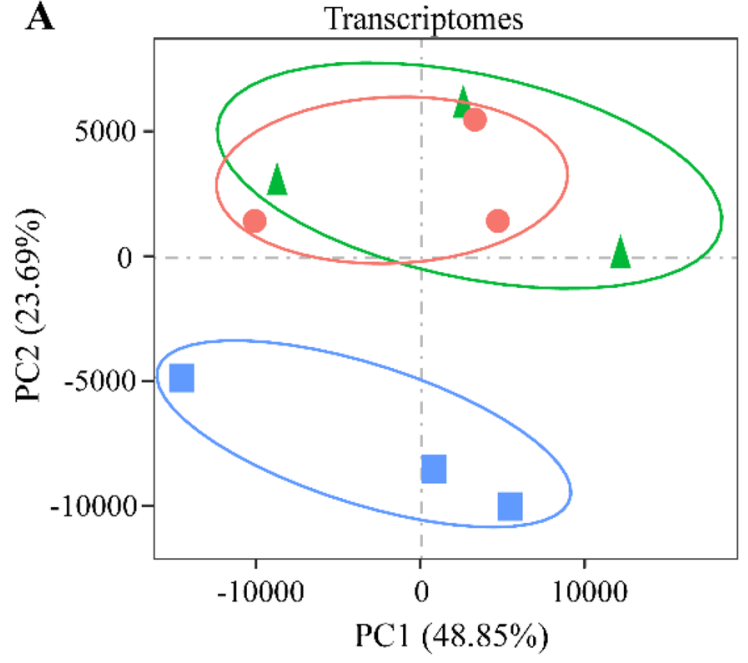

C

Transcriptomes

Event- 2.4 vs Event- $3.5 \quad$ Event- 2.4 vs CK

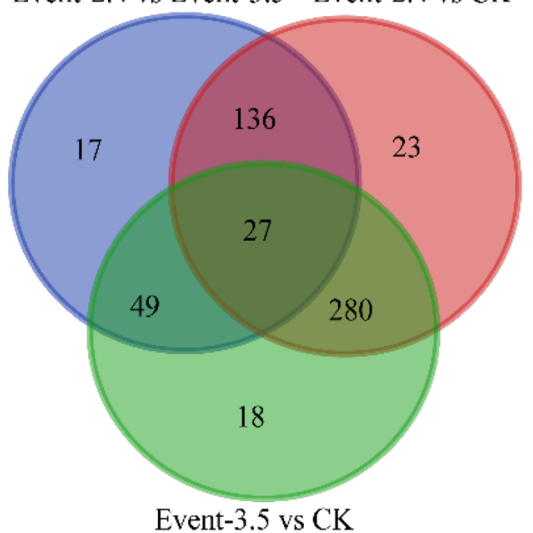

$\mathbf{E}$

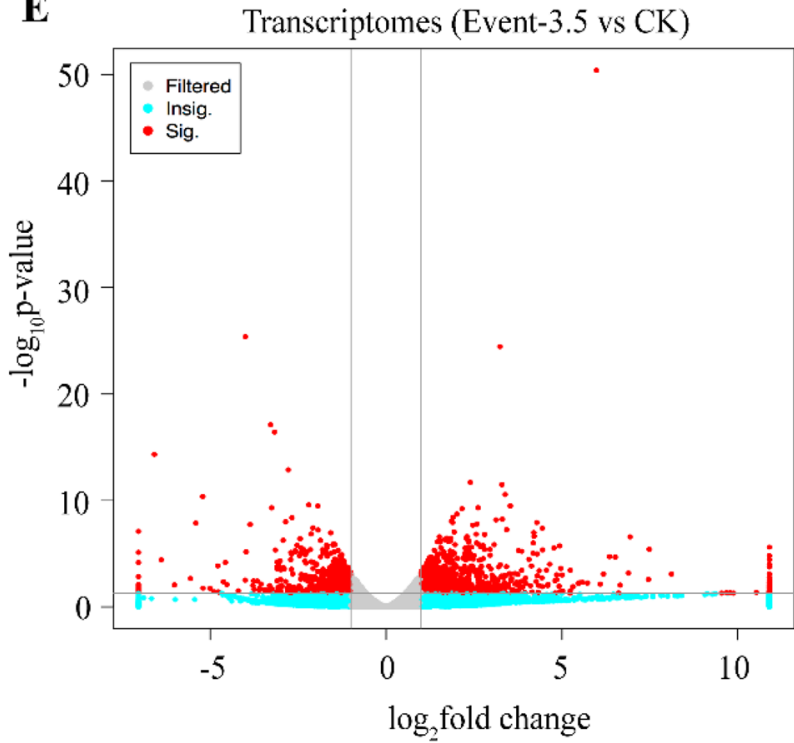

B

D

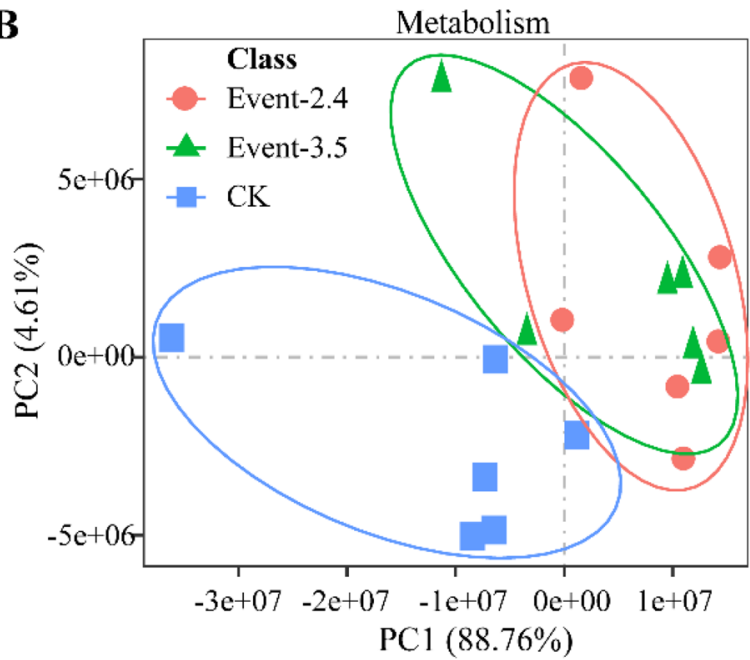

Metabolism

Event-2.4 vs Event-3.5 Event-2.4 vs CK

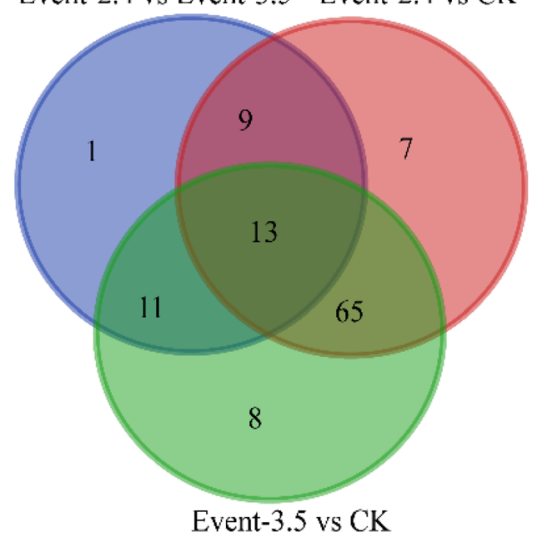

$\mathbf{F}$
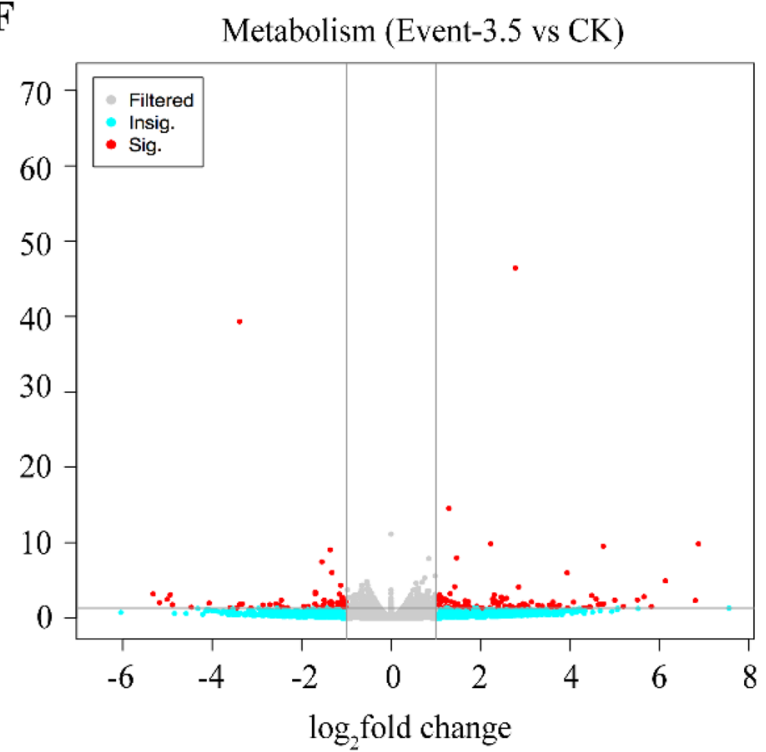

Figure 3. PCA analysis on differently abundant genes and metabolites in ZD958 maize transgenic lines grown in Beijing. (A), transcriptomes analysis on different experimental sites across different transgenic lines of transgenic event-2.4 (Event-2.4), transgenic event-3.5 (Event-3.5) and $\mathrm{CK} ; \mathrm{n}=3$. $(\mathrm{B})$, metabolomic analysis on different experimental sites across different transgenic lines of 2.4, 3.5 and ck. $n=6$. $(\mathbf{C})$, overlapping genes between different ZD958 transgenic events and non-GM ZD958 (CK) grown in Beijing. The detailed overlapping genes are referred to Supplementary Table S2. (D), overlapping metabolites. The detailed overlapping metabolites are referred to Supplementary Table S3. (E,F), differential abundant genes and metabolites between event-3.5 and CK. The differential abundant genes (DAGs) and metabolites (DAMs) were indicated in red scatters. 


\begin{tabular}{|c|c|c|c|c|}
\hline Ensembl gene id & GM-ZD958-2.4 & GM-ZD958-3.5 & $p$-value & Gene description \\
\hline Zm00001d053936 & 3.21 & 2.78 & $8.84 \mathrm{E}-06$ & $\begin{array}{l}\text { Polyketide cyclase/dehydrase and lipid transport superfamily } \\
\text { protein }\end{array}$ \\
\hline $\mathrm{Zm} 00001 \mathrm{~d} 014108$ & 3.17 & 3.27 & $8.62 \mathrm{E}-06$ & Ubiquitin-conjugating enzyme E2 4 \\
\hline Zm00001d028951 & 3.78 & 3.65 & $3.33 \mathrm{E}-07$ & Small basic membrane intrinsic protein $2 \mathrm{a}$ \\
\hline $\mathrm{Zm00001d014944}$ & 6.96 & 2.28 & $4.72 \mathrm{E}-06$ & Lactate dehydrogenase 1 \\
\hline Zm00001d001911 & 5.20 & 5.37 & $6.20 \mathrm{E}-07$ & ATP-dependent RNA helicase \\
\hline Zm00001d038922 & 2.23 & 2.32 & $2.98 \mathrm{E}-06$ & CRS1/YhbY (CRM) domain-containing protein \\
\hline $\mathrm{Zm} 00001 \mathrm{~d} 012446$ & 5.13 & 4.78 & $5.45 \mathrm{E}-06$ & V-type proton ATPase subunit a1 \\
\hline Zm00001d021635 & 6.16 & 6.25 & $1.85 \mathrm{E}-05$ & Condensin- 2 complex subunit $\mathrm{H} 2$ \\
\hline $\mathrm{Zm00001d016477}$ & 7.61 & 7.83 & $1.90 \mathrm{E}-06$ & Eukaryotic aspartyl protease family protein \\
\hline $\mathrm{Zm} 00001 \mathrm{~d} 034717$ & 6.93 & 6.59 & $2.88 \mathrm{E}-06$ & $\begin{array}{l}\text { S-Adenosyl-L-methionine-dependent methyltransferases super- } \\
\text { family protein }\end{array}$ \\
\hline Zm00001d009709 & 6.06 & 6.63 & $1.33 \mathrm{E}-06$ & Loricrin-related \\
\hline $\mathrm{Zm} 00001 \mathrm{~d} 013358$ & 2.58 & 2.91 & $8.79 \mathrm{E}-06$ & DEAD-box ATP-dependent RNA helicase 16 \\
\hline $\mathrm{Zm} 00001 \mathrm{~d} 037840$ & 5.03 & 5.87 & $6.09 \mathrm{E}-06$ & $\begin{array}{l}\text { Putative leucine-rich repeat receptor-like protein kinase family } \\
\text { protein }\end{array}$ \\
\hline Zm00001d036571 & 2.22 & 2.57 & $4.22 \mathrm{E}-06$ & Heat shock $70 \mathrm{kDa}$ protein 16 \\
\hline $\mathrm{Zm00001d047502}$ & 3.96 & 4.22 & $2.92 \mathrm{E}-06$ & DEAD-box ATP-dependent RNA helicase 35 \\
\hline $\mathrm{Zm} 00001 \mathrm{~d} 015130$ & 3.54 & 3.59 & $1.22 \mathrm{E}-05$ & SBP (S-ribonuclease binding protein) family protein \\
\hline Zm00001d044451 & 4.07 & 4.14 & $2.91 \mathrm{E}-06$ & RING/FYVE/PHD zinc finger superfamily protein \\
\hline
\end{tabular}

Table 2. Fold change of top 5\% differentially abundant genes (DAGs) between two transgenic events of GM-ZD958 relative to non-GM ZD958 (CK).

\begin{tabular}{|l|l|l|l|l|l|l|l|}
\hline Metabolites & Molecular weight & Retention time $(\mathbf{m i n})$ & GM-ZD958-2.4 & GM-ZD958-3.5 & Z58 & Chang7-2 & p-value \\
\hline Galacturonic acid & 236.465 & 8.3 & 20.727 & 22.860 & 1.036 & 1.095 & $2.30 \mathrm{E}-05$ \\
\hline L-Asaparagine & 228.842 & 11.678 & 18.316 & 19.997 & 1.039 & 0.992 & $2.8 \mathrm{E}-07$ \\
\hline
\end{tabular}

Table 3. Fold change for top 5\% DAM in HIR gene cassette GM-ZD958 transgenic lines and its nonGM parents (Z58 and Chang7-2), relative to nonGM-ZD958 determined in growth chamber. $P$-value represent the significant levels performed by one-way ANOVA among different maize lines.

ing HIR gene cassette and original transgenic background for both Z58 and ZD958 regardless of transcriptomes or metabolisms (Fig. 8A,B).

\section{Discussion}

With the developments of GM, a variety of transgenic maize plants have been produced with different characteristics including insect resistant-maize and herbicide-tolerant maize. In this study, we introduced a HIR gene cassette, which confers herbicide and insect resistance, into a commercial maize variety, Zhengdan958 (ZD958). We presented the first multi-omic analysis of GM-ZD958 maize compared to a non-GM ZD958 maize line. Our analysis at a detailed, in-depth molecular profiling levels shows that marginal effects on the alteration of differential abundant genes and metabolites in GM-ZD958, compared to its non-GM ZD958 maize line.

Case-study on transgenic events effects on molecular profiling. Unintended effects of GM crops are the main concern of administrators and citizens after their intended effects have been thoroughly investigated. This unintended effects majorly derived from random integration on undesirable genomic-regions during transgenic process such as agrobacteria-mediated transformation ${ }^{9}$. We present the first time transgenic events effects on molecular profiling. Two-independent transgenic events (event-2.4, and event-3.5) were applied, through using same gene constructs to elucidate differential abundant transcripts and metabolites due to transgenic effects and off-target effects (Fig. 3). We identified only $0.5 \%$ DAGs that observed between event-2.4 and event-3.5 (Fig. 3C). Interestingly, the numbers of DAGs induced by HIR gene cassette effects compared to nonGM ZD958 (CK) account 5\% for total annotated genes. In addition, only 17 genes showing no significant difference between two transgenic events but showing significant difference compared to CK (Table 2). However, no significant biological pathways or categories were enriched. We found that around $4 \%$ differentially abundant metabolites (DAMs) account for total identified metabolites due to HIR gene cassette effects irrespective of offtarget effects, which is in line with similar numbers of metabolites as observed in NK603 maize lines ${ }^{16}$. These findings suggest that the off-target and gene effects are equivalent in the derived maize lines from two transgenic integration events HIR gene cassette effects. 


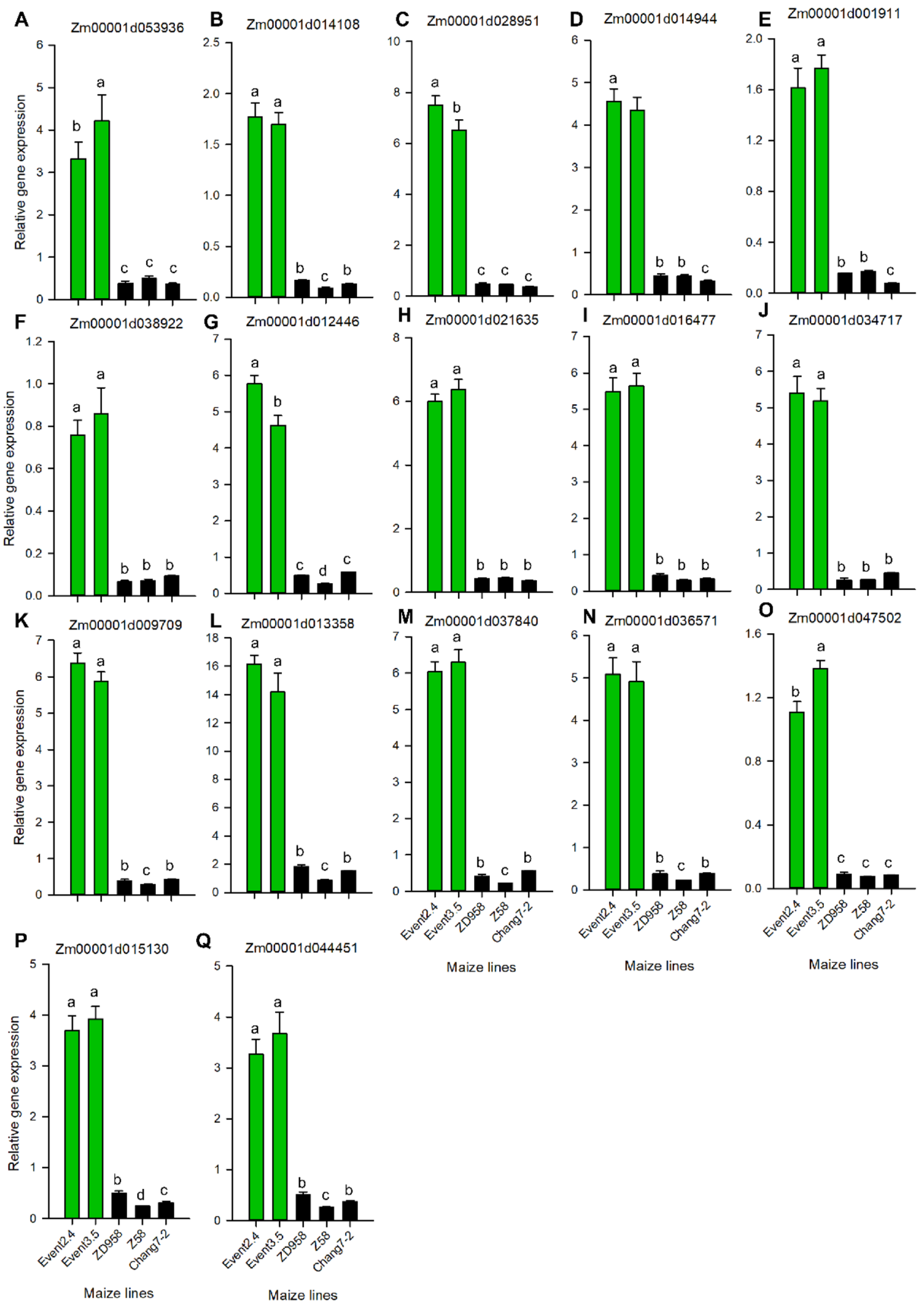

Figure 4. qPCR testing the relative gene expression with top 5\% differential expressed genes between two transgenic event (3.5 and 2.4) and other genetic background (Chang7-2, Z58) and non-GM counterpart (ZD958) (A-Q). Different alphabet letters represent significant differences at $P<0.05$ based on one-way ANOVA. $n=6$. 
A

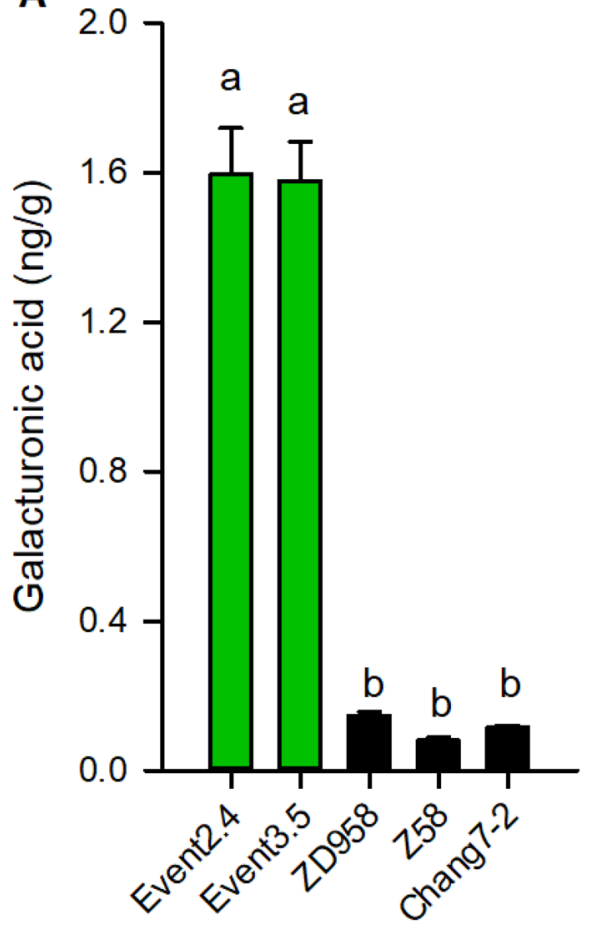

Maize lines
B

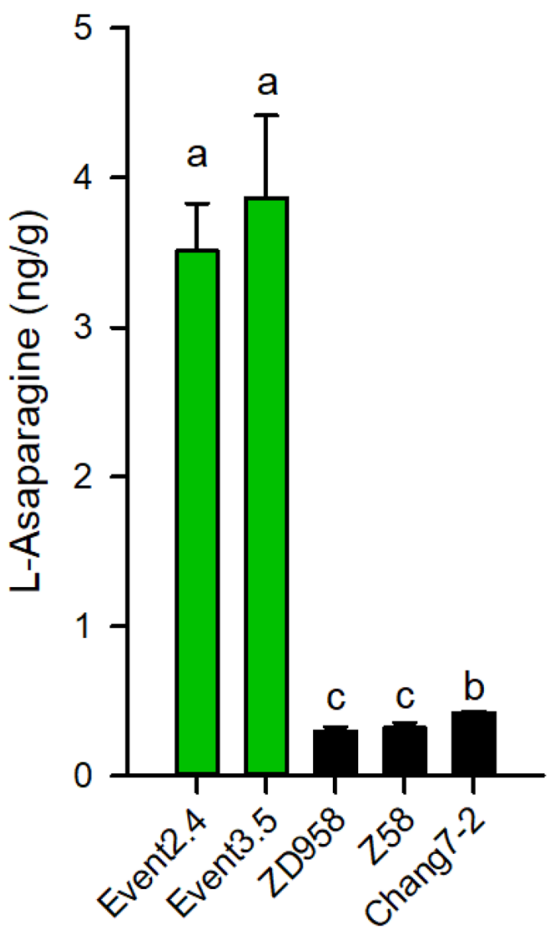

Maize lines

Figure 5. Targeted metabolites measurement to confirm the contents changes induced by HIR gene cassette effects using LC-MS/MS with standard curve (a-b). Different alphabet letters represent significant differences at $P<0.05$ based on one-way ANOVA. $n=6$.

Reprogrammed molecular profiling induced by environmental effects. We looked at the combined effects of genetic modification and growing environments by planting two GM-ZD958 across two transgenic event (event 2.4 and 3.5) and non-GM ZD958) in three different ecological regions of China, i.e., Harbin, Zhengzhou, and Beijing. A distinct separation between Harbin with other two ecological regions was observed, however partial overlapping was observed between Zhengzhou and Beijing for both transcriptomics and metabolic analysis. This is very like due to the difference of average surface air temperature during growth season (May to Oct) between three sites, Harbin $\left(17^{\circ} \mathrm{C}\right)$, Beijing $\left(28^{\circ} \mathrm{C}\right)$, and Zhengzhou $\left(30^{\circ} \mathrm{C}\right)$. This geo-climate distribution reprograms maize transcriptome, proteome, and metabolome and even by activating cell death mechanisms leading to organ abortion or entire plant death ${ }^{17,18}$. Indeed, we found 894 up-regulated genes due to relatively low temperature in Harbin compared to normal temperature recorded in Zhengzhou. Among these genes, we found they are significantly enriched in the pathway relating to plants response to abiotic tress, including phosphorelay signal transduction system and Arginine/proline metabolism according to GO and KEGG analysis (Fig. 7A,B). Under low temperature environments, integration of cellular membrane was injured, which leads to overproduction of $\operatorname{ROS}^{19}$. As protective mechanism, proline, a major type of antioxidant is accumulated, to remove ROS under low temperature condition ${ }^{20}$. Meanwhile, plants evolve to "switch on" the adaptive response through using this phosphorelay signaling machineries, which enables plants to confers tolerance toward abiotic stress $^{21}$. These findings suggest that the environment was even shown to play a stronger effect in gene expression, and metabolite levels of the GM samples than gene modification by previous publications, which showed similar results for transcriptomics in maize and other species, such as rice, wheat, soybean, and potato ${ }^{13,22-26}$.

Greater genetic background effect on molecular profiling than gene effects. Many efforts have been made to test if GM crops are substantially equivalent as safe non-GM comparators, following a large body of high-quality compositional data that need to be determined according to principles outlined in the Organization for Economic Cooperation and Development ${ }^{27}$. Our study design further highlights the importance of restricting comparison to the two GM maize (GM-ZD958 and GM-Z58) and two non-GM maize lines (nonGMZD958 and nonGM-Z58) and cultivation of the two at the same location and growing environments, when the objective is to evaluate the effect of the GM transformation process. A closed cluster regarding transcriptomes and metabolism in ZD958 maize varieties harboring HIR gene cassette and its non-GM ZD958 was observed, while it apart from another background nonGM-Z58 (Fig. 8A,B). This suggests that the molecular variability such as gene expression and metabolites levels in maize varieties harboring HIR gene cassette are relatively closed, but yielding different with non-GM comparators. As mentioned above, the gene expression and metabo- 
A

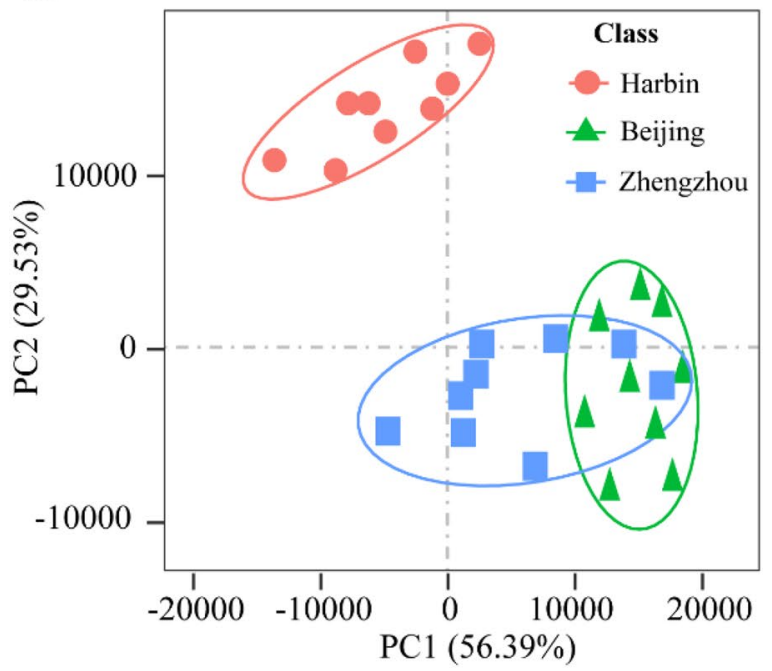

C

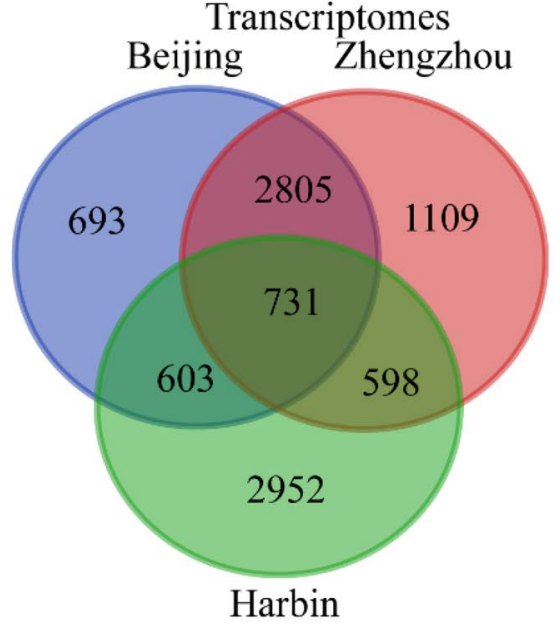

E

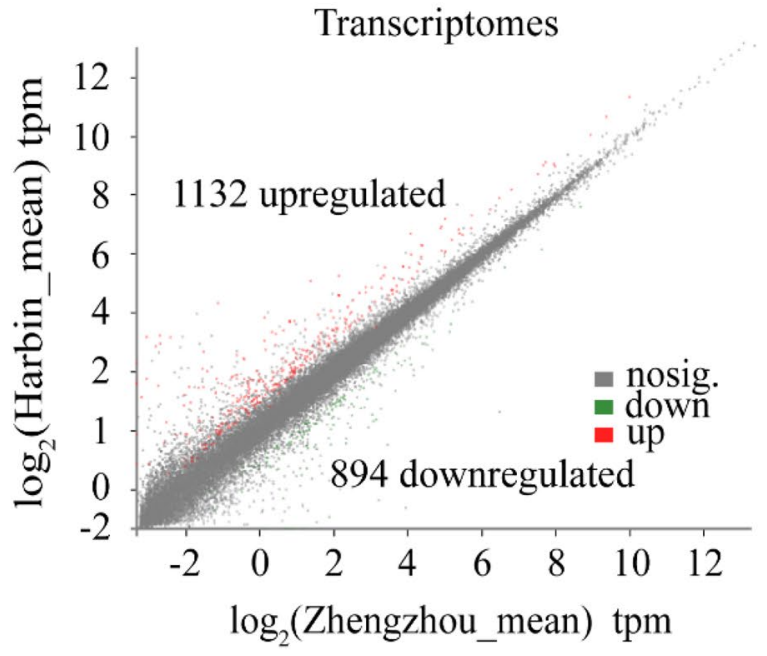

B

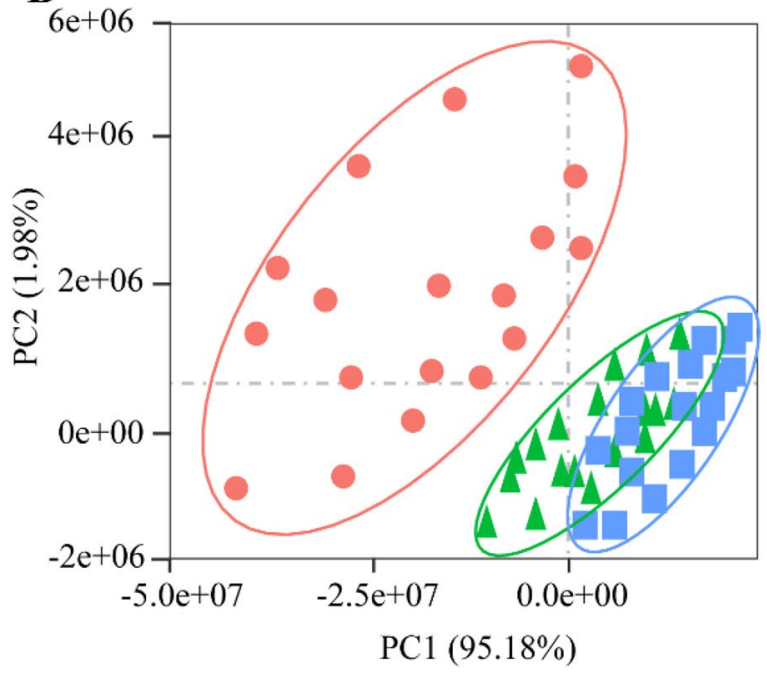

D

Metabolisms

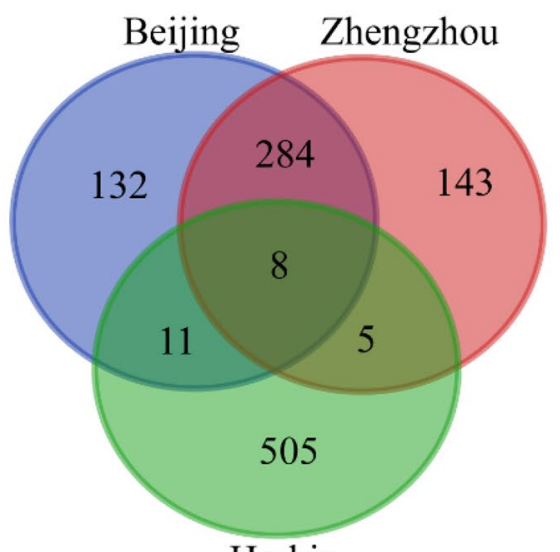

Harbin

\section{F}

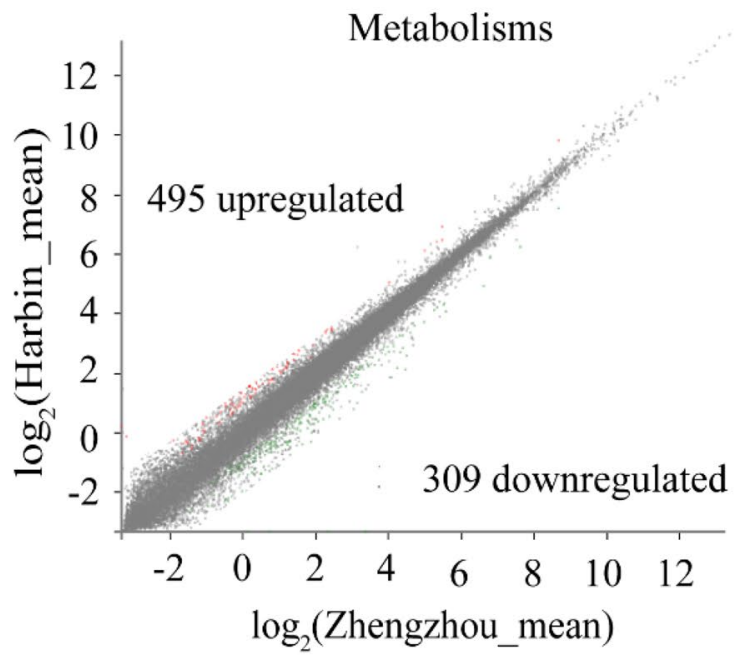

Figure 6. Principal component analysis on different experimental sites for GM-ZD958 across two different transgenic event lines (event-2.4 and 3.5) and non-GM ZD958 (CK). (A) Transcriptomes analysis on different experimental sites, i.e., Beijing $\left(\mathrm{E} 116.3^{\circ}, \mathrm{N} 39.7^{\circ}\right)$, Harbin $\left(\mathrm{E} 125.4^{\circ}, \mathrm{N} 44.1^{\circ}\right)$, and Zhengzhou $\left(\mathrm{E} 113.6^{\circ}, \mathrm{N} 34.7^{\circ}\right)$ of China, across different transgenic events and CK; $n=9$. (B) Metabolomics analysis on different experimental sites across different transgenic events and CK. $n=18$. (C,D), Effects of different growing environments on differentially abundant genes or metabolites. (E,F), Comparison on down-/up-regulation of genes or metabolites between Zhengzhou and Harbin. 
A

GO analysis

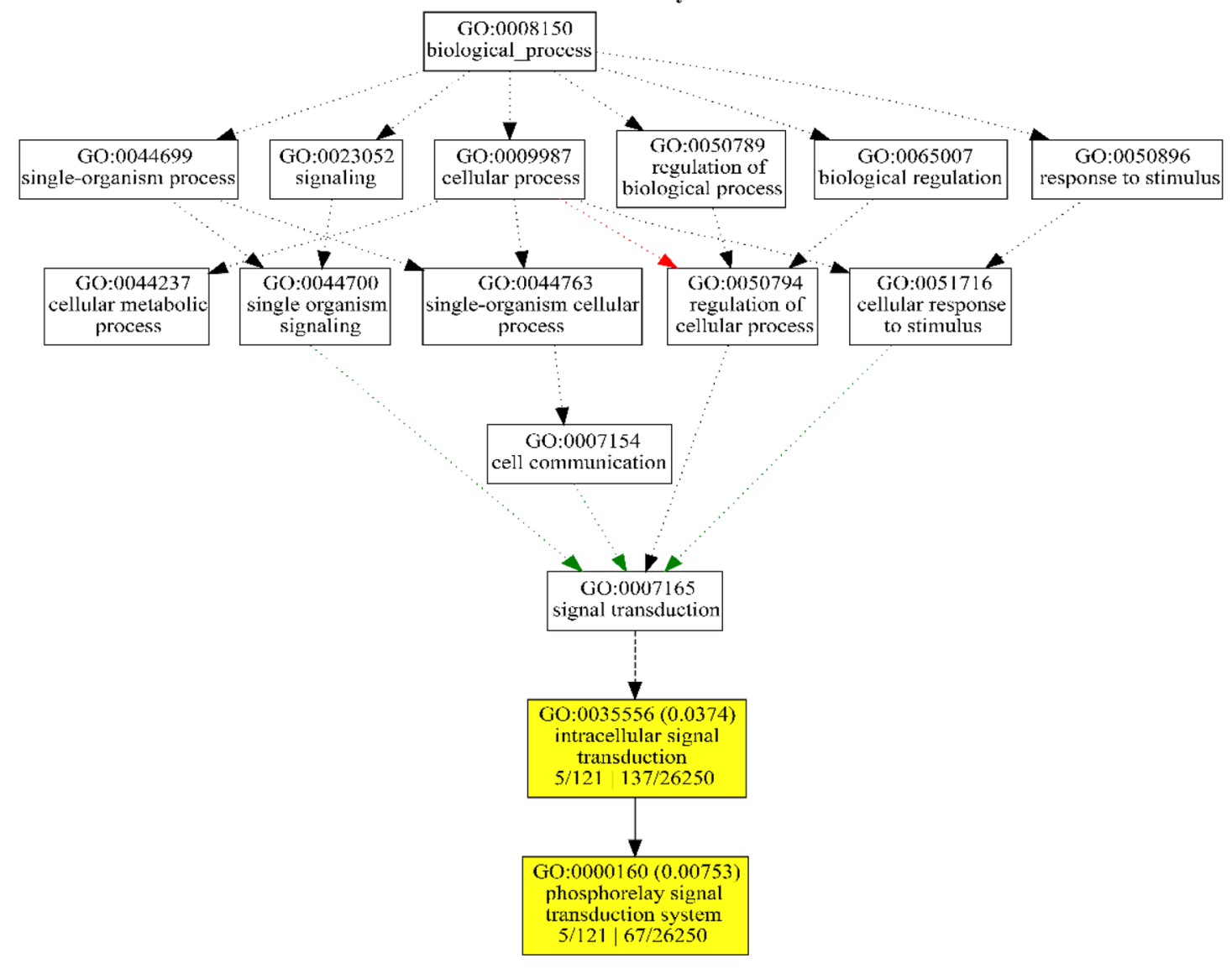

B

KEGG analysis

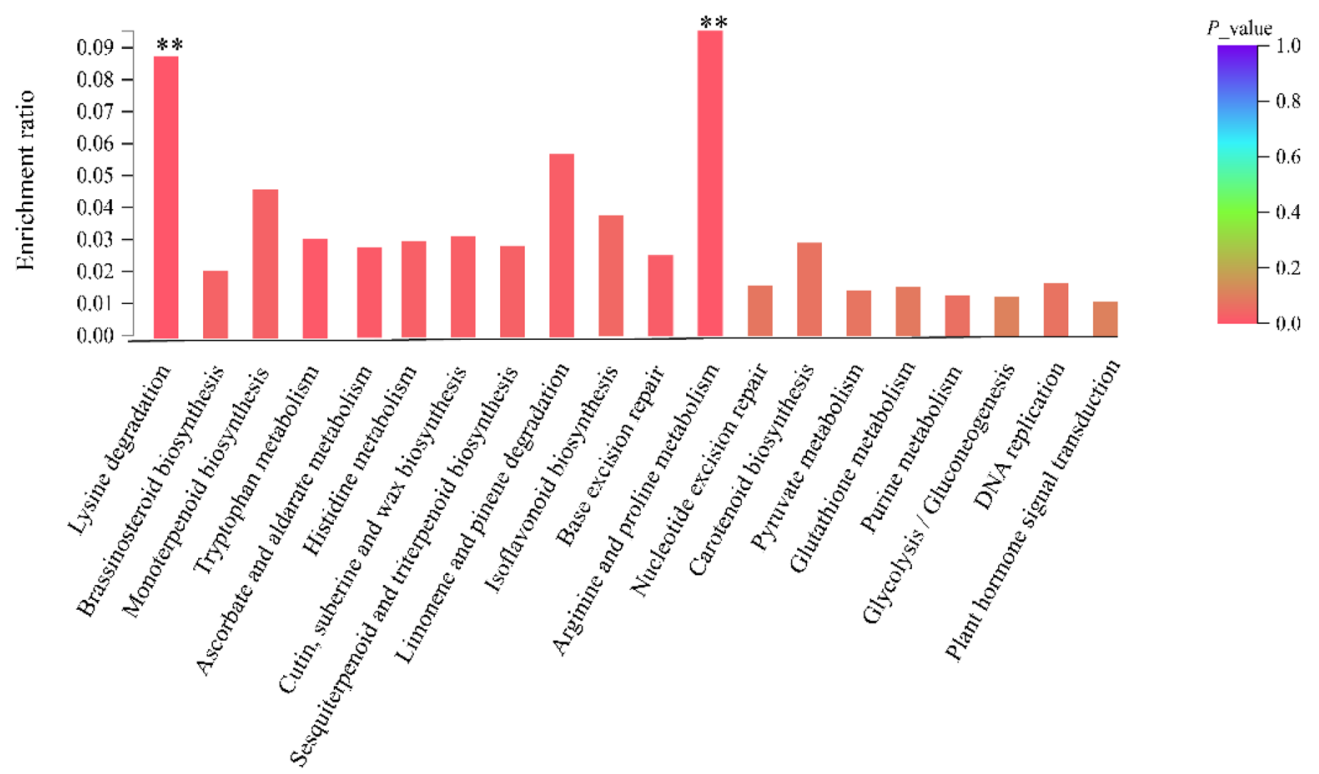

Figure 7. Bioinformatic analysis on different abundant genes in maize grown in Harbin compared to Zhengzhou. The abundance of genes derived from the average across CK and two transgenic events in GM-ZD958 maize lines. (A) Gene Ontology (GO) analysis on different abundant genes (DAGs) in maize grown in Harbin compared to Zhengzhou. Enriched biological pathway were highlighted in yellow. (B) Kyoto Encyclopedia of Genes and Genomes (KEGG) analysis on DAGs in maize grown in Harbin compared to Zhengzhou. Significantly enriched metabolic pathways were shown symbol " "” and " "**”, as indicated $P$ value $<0.05$ and 0.01 , respectively. 
A

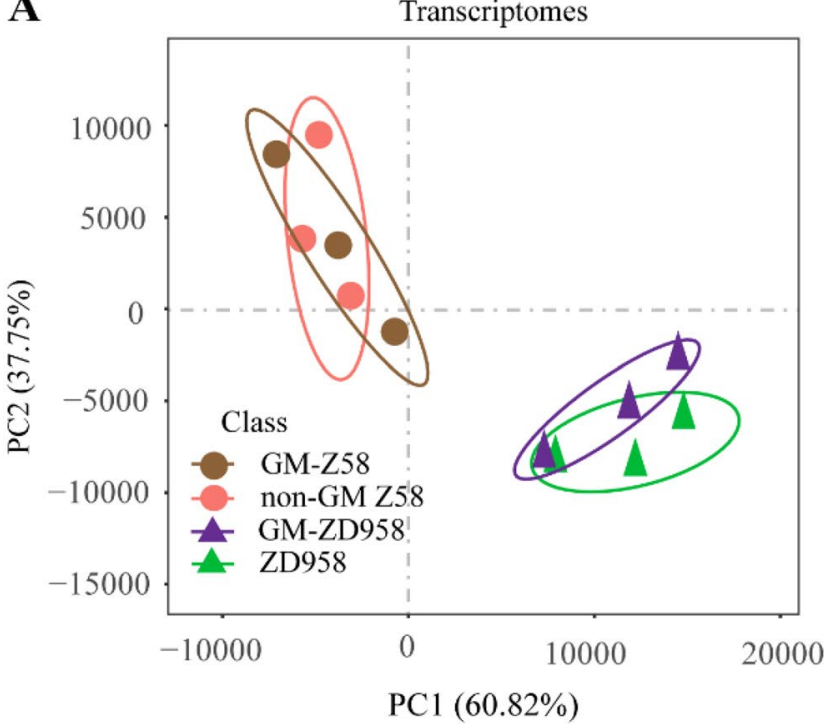

B

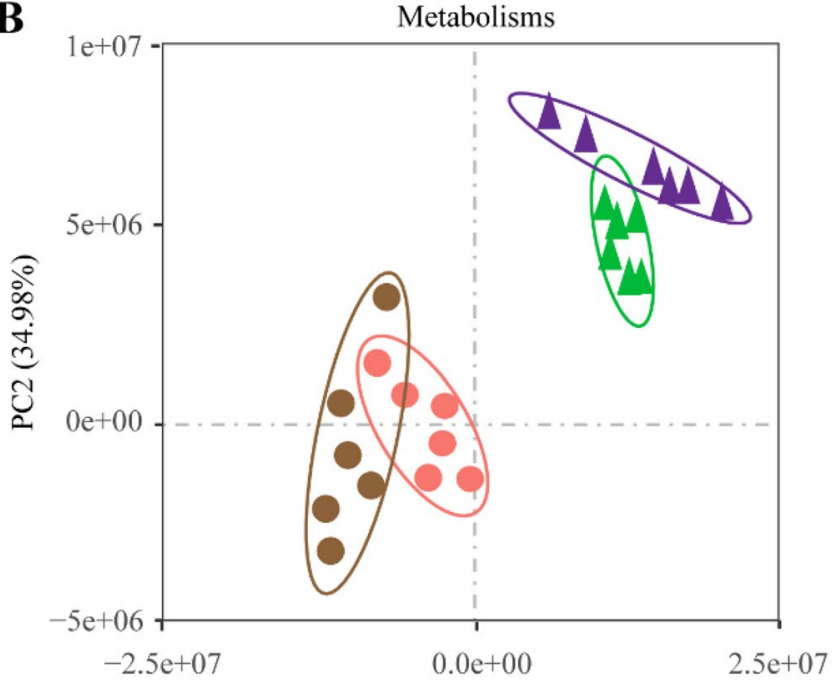

PC1 (62.95\%)

Figure 8. Principal component analysis on GM-ZD958 maize transgenic lines across two transgenic events against different genetic background GM-Z58, Z58, and non-GM ZD958 grown under growth chamber across two transgenic events. (A) transcriptomes data; (B) metabolic data.

lites were largely altered by growing environments (Fig. 6A,B). We extracted the list of genes and metabolites with no significant differences among three growing environments for each GM/or non-GM maize variety, to minimize the environmental effects on the following analysis.

\section{Materials and methods}

Plant material and plant growth. In this study, Zhengdan58 (ZD958) is used as a reference hybrid line, representing maize lines in F1 generation from its parent inbred lines, i.e., Zheng58 and Chang7-2. The two parent inbred lines are gifts from Beijing Dabeinong Biotechnology Co., Ltd.. Meanwhile, the GM-ZD958 maize cultivar used in this study were derived from a non-GM inbred line Change 7-2 and a transgenic receptor, Zheng58, by expressing a HIR gene cassette suite which consists of two genes, i.e., EPSPS and Cry1Ab, responsible for the transgenic herbicide (glyphosate) and insect (Bacillus thuringiensis) resistance genes ${ }^{28,29}$, respectively. For transgenic ZD958 hybrid line, we conducted two independent transgenic events (3.5 and 2.4) from the same construct harboring the HIR gene cassette, to eliminate the random insertion effects on undesirable genomic regions. The plants were grown in the field in three different ecological regions of China, namely, Beijing, Zhengzhou, and Harbin, as well as indoor chambers in Beijing as shown in Table 1.

RNA extraction and transcriptomes analysis. Total RNA was extracted from the leaves of 40 days old maize lines using TRIzol Reagent according to manufacturer's instructions (Invitrogen, Ohio, USA) ${ }^{30}$. Genomic DNA was removed using DNase I kit (TaKara, Tokyo, Japan). Quality and purity of RNA were determined by $1 \%$ of agarose gels and nano-drop (IMPLEN, California, USA), respectively. RNA-seq transcriptome library was constructed using around $5 \mu \mathrm{g}$ of total RNA based on NEBNext Ultra RNA Kit (Illumina, San Diego, CA, USA) ${ }^{31}$. Briefly, mRNA was firstly isolated through oligo (dT) beads, and crushed by fragmentation buffer, and then double-stranded cDNA was synthesized using a SuperScript double-stranded cDNA synthesis kit (Invitrogen, CA, USA). Then the synthesized cDNA was subjected to end-repair, phosphorylation and 'A' base addition according to Illumina's library construction protocol. Libraries were size selected for cDNA target fragments of 200-300 bp on 2\% Low Range Ultra Agarose followed by PCR amplified using Phusion DNA polymerase (NEB) for 15 PCR cycles. After quantification, paired-end RNA-seq sequencing library was sequenced with the Illumina HiSeq $4000(2 \times 150 \text { bp read length })^{31}$.

The resulting paired-end reads were trimmed and merged with SeqPrep (https://github.com/jstjohn/SeqPr ep). The trimming Illumina paired end reads were quality controlled by Sickle (https://github.com/najoshi/sickl e). Then clean reads were separately aligned to reference genome with orientation mode using TopHat (http:// tophat.cbcb.umd.edu/, version 2.0.0) software ${ }^{32}$. The mapping criteria of bowtie was as follows: sequencing reads should be uniquely matched to the genome allowing up to 2 mismatches, without insertions or deletions. Then, the regions of gene were expanded following depths of sites and the operon was obtained. In addition, the whole genome was split into multiple $15,000 \mathrm{bp}$ windows that share $5000 \mathrm{bp}$. Therefore, the new transcribed regions were defined as more than 2 consecutive windows without overlapped region of gene, where at least 2 reads mapped per window in the same orientation.

To identify differentially abundant genes (DAGs) between two different samples, the expression level of each transcript was calculated according to Transcript per million (TPM) method, which considers the gene length for normalization, and is suitable for sequencing protocols where reads sequencing depends on gene length ${ }^{33}$. RSEM (http://deweylab.biostat.wisc.edu/rsem/) was used to quantify gene abundance ${ }^{34}$. R statistical package software 
EdgeR (Empirical analysis of Digital Gene Expression in R, http://www.bioconductor.org/ packages/2.12/bioc/ html/edgeR.html) was utilized for differential expression analysis ${ }^{35}$.

GO and KEGG analysis. An in-house Perl script was used to perform gene ontologies (GO) annotation based on UniProtKB GOA file (ftp.ebi.ac.uk/pub/databases/GO/goa/UNIPROT/gene_association.goa_uniprot. gz). KOBAS (KEGG Orthology Based Annotation System, v2.0) was used to identify biochemical pathways and to calculate the statistical significance for each pathway ${ }^{36}$.

Quantitative real time PCR. Real-time PCR (qPCR) was used to validate the differentially abundant genes in different background of maize varieties. Total RNA from maize leaves was isolated using Ultra-Pure RNA Kit (cwbiotech), and complementary DNA was synthesized with ExScript RT Kit (Takara). The amplification reaction conditions were as follows: $95^{\circ} \mathrm{C}$ for $3 \mathrm{~min}$ followed by 40 cycles of $95^{\circ} \mathrm{C}$ for $10 \mathrm{~s}$ and $60^{\circ} \mathrm{C}$ for $30 \mathrm{~s}$. The primer is listed in Table S1. Actin gene was used as internal control. The relative gene expression level was calculated based on $2^{-\Delta \Delta \mathrm{CT}}$ as described previously ${ }^{37}$. Three biological replicates were performed.

LC-MS/MS based metabolism and identification. Non-targeted metabolic profiling in different transgenic maize lines were determined based on LC-MS/MS (Q Exactive, Thermo Scientific). $2.5 \mathrm{mg}$ leaves of 30 days-old maize were sampled in $2 \mathrm{~mL}$ Eppendorf tube filled with pre-cooled metal beads, and immediately stored in liquid nitrogen. The samples were first extracted with ball mill at $30 \mathrm{~Hz}$ for $5 \mathrm{~min}$, and the extracted powder was then dissolved with $1.5 \mathrm{~mL}$ methanol/chloroform, and incubated at $-20{ }^{\circ} \mathrm{C}$ for $5 \mathrm{~h}$. The mixture was centrifuged at $2000 \mathrm{~g}$ at $4{ }^{\circ} \mathrm{C}$ for $10 \mathrm{~min}$, and then was filtered with $0.43 \mu \mathrm{m}$ organic phase medium (GE Healthcare, 6789-0404). The metabolomics profiling analysis was performed by Metabolon software (Durham, NC, USA). For two identified metabolites, i.e., galacturonic acid and L-asaparagine, the contents of two metabolites were further determined by LC-MS/MS (AB Sciex Qtrap 6,500; SCIEX) with standard curve as described previously $^{38}$. Metabolites were identified by analyzing retention times and mass spectra with those for reference compounds as well as the entries of the mass spectra libraries NIST02 and Golm metabolome database (http:// csbdb.mpimp-golm.mpg.de/csbdb/gmd/gmd.html).

Data analysis. Principal component analysis (PCA) was performed using SYSTAT 11 (Systat Software Inc., CA, USA $)^{39}$. One-way ANOVA was performed in combination with modified Tukey's HSD test to identify the differences in the relative abundance of a given transcript, and the metabolites. Differences at the level $P<0.01$ were considered statistically significant.

\section{Data availability}

All data is available in the manuscript or the supplementary materials.

Received: 9 February 2020; Accepted: 16 December 2020

Published online: 21 January 2021

\section{References}

1. Pellegrino, E., Bedini, S., Nuti, M. \& Ercoli, L. Impact of genetically engineered maize on agronomic, environmental and toxicological traits: A meta-analysis of 21 years of field data. Sci. Rep. 8, 3113 (2018).

2. Huang, J., Rozelle, S., Pray, C. \& Wang, Q. Plant biotechnology in China. Science 295, 674-677 (2002).

3. Baker, J. M. et al. A metabolomics study of substantial equivalence of field-grown genetically modified wheat. Plant Biotechnol. J. 4, 381-392 (2006)

4. Kok, E. J. \& Kuiper, H. A. Comparative safety assessment for biotech crops. Trends Biotechnol. 21, 439-444 (2003).

5. National Academies of Sciences E, Medicine. Genetically Engineered Crops: Experiences and Prospects. (National Academies Press, Washington, DC, 2017).

6. Kuiper, H. A., Kleter, G. A., Notenorn, H. O. \& Kok, E. J. Assessment of the food safety issues related to genetically modified foods. Plant J. 27, 503-528 (2001).

7. Cellini, F. et al. Unintended effects and their detection in genetically modified crops. Food Chem. Toxicol. 42, 1089-1125 (2004).

8. Gould, J. et al. Transformation of Zea mays L. using Agrobacterium tumefaciens and the shoot apex. Plant Physiol. 95, 426-434 (1991).

9. Vain, P., McMullen, M. D. \& Finer, J. J. Osmotic treatment enhances particle bombardment-mediated transient and stable transformation of maize. Plant Cell Rep. 12, 84-88 (1993).

10. Kim, H. A. et al. The development of herbicide-resistant maize: Stable Agrobacterium mediated transformation of maize using explants of type II embryogenic calli. Plant Biotechnol. Rep. 3, 277-283 (2009).

11. Strauss, S. H. \& Sax, J. K. Ending event-based regulation of GMO crops. Nat. Biotechnol. 34, 474-477 (2016).

12. Fu, W., Wang, C., Xu, W. \& Zhu, P. Unintended effects of transgenic rice revealed by transcriptome and metabolism. GM Crops Food 10, 20-34 (2019).

13. Barros, E. et al. Comparison of two GM maize varieties with a near-isogenic non-GM variety using transcriptomics, proteomics and metabolomics. Plant Biotechnol. J. 8, 436-451 (2010).

14. Pauwels, K. et al. Next generation sequencing as a tool for the molecular characterization and risk assessment of genetically modified plants: Added value or not?. Trends Food Sci. Technol. 45, 319-326 (2015).

15. Benbrook, C. M. Impacts of genetically engineered crops on pesticide use in the U.S.-the first sixteen years. Environ. Sci. Eur. 24, 24 (2012).

16. Mesnage, R. et al. An integrated multi-omics analysis of the NK603 Roundup-tolerant GM maize reveals metabolism disturbances caused by the transformation process. Sci. Rep. 6, 37855 (2016).

17. Qi, Y. et al. Over-expression of mitochondrial heat shock protein 70 suppresses programmed cell death in rice. FEBS Lett. 2585, 231-239 (2011).

18. Sánchez-Rodríguez, E., Moreno, D. A., Ferreres, F., Rubio-Wilhelmi, M. \& Ruiz, J. M. Differential responses of five cherry tomato varieties to water stress: Changes on phenolic metabolites and related enzymes. Phytochemistry 72, 723-729 (2011). 
19. Dreyer, A. \& Dietz, K. J. Reactive oxygen species and the redox-regulatory network in cold stress acclimation. Antioxidants (Basel) 7, 169 (2018).

20. Ignatenko, A., Talanova, V., Repkina, N. \& Titov, A. Exogenous salicylic acid treatment induces cold tolerance in wheat through promotion of antioxidant enzyme activity and proline accumulation. Acta Physiol. Plant. 41, 80 (2019).

21. Nongpiur, R., Soni, P., Karan, R., Singla-Pareek, S. L. \& Pareek, A. Histidine kinases in plants: Cross talk between hormone and stress responses. Plant Signal Behav. 7, 1230-1237 (2012).

22. Cheng, K. et al. Effect of transgenes on global gene expression in soybean is within the natural range of variation of conventional cultivars. J. Agric. Food Chem. 56, 3057-3067 (2008).

23. Batista, R., Saibo, N., Lourenço, T. \& Oliveira, M. M. Microarray analyses reveal that plant mutagenesis may induce more transcriptomic changes than transgene insertion. Proc. Natl. Acad. Sci. U. S. A. 105, 3640-3645 (2008).

24. Baudo, M. M. et al. Transgenesis has less impact on the transcriptome of wheat grain than conventional breeding. Plant Biotechnol. J. 4, 369-380 (2006).

25. Kogel, K. H. et al. Transcriptome and metabolome profiling of field-grown transgenic barley lack induced differences but show cultivar-specific variances. Proc. Natl. Acad. Sci. U. S. A. 107, 6198-6203 (2010).

26. Catchpole, G. S. et al. Hierarchical metabolomics demonstrates substantial compositional similarity between genetically modified and conventional potato crops. Proc. Natl. Acad. Sci. U. S. A. 102, 14458-14462 (2005)

27. OECD. An introduction to the food/feed safety consensus documents of the task force. Ser. Saf. Novel Foods Feeds 14, 7-9 (2006).

28. $\mathrm{Hu}$, Y. et al. Three-generation reproductive toxicity of genetically modified maize with Cryl Ab and epsps genes. J. Agric. Food Chem. https://doi.org/10.1021/acs.jafc.0c02237 (2020).

29. Liu, W., Liang, L., Zhang, Z., Dong, M. \& Jin, W. iTRAQ-based quantitative proteomic analysis of transgenic and non-transgenic maize seeds. J. Food Compost. Anal. 92, 103564 (2020).

30. Li, J. et al. Roles of heat shock protein and reprogramming of photosynthetic carbon metabolism in thermotolerance under elevated $\mathrm{CO}_{2}$ in maize. Environ. Exp. Bot. 168, 103869 (2019).

31. Essemine, J., Li, J., Chen, G. \& Qu, M. Analytical dataset of short-term heat stress induced reshuffling of metabolism and transcriptomes in maize grown under elevated $\mathrm{CO}_{2}$. Data Brief https://doi.org/10.1016/j.dib.2019.105004 (2019).

32. Trapnell, C., Pachter, L. \& Salzberg, S. T. TopHat: Discovering splice junctions with RNA-Seq. Bioinformatics 25, 1105-1111 (2009).

33. Wagner, G. P., Kin, K. \& Lynch, V. J. Measurement of mRNA abundance using RNA-seq data: RPKM measure is inconsistent among samples. Theory Biosci. 31, 281-285 (2012).

34. Li, B., Ruotti, V., Stewart, R. M., Thomson, J. A. \& Dewey, C. M. RNA-Seq gene expression estimation with read mapping uncertainty. Bioinformatics 26, 493-500 (2010).

35. Robinson, M. D., McCarthy, D. J. \& Smyth, G. K. edgeR: A bioconductor package for differential expression analysis of digital gene expression data. Bioinformatics 26, 139-140 (2010).

36. Xie, C. et al. KOBAS 2.0: A web server for annotation and identification of enriched pathways and diseases. Nucleic Acids Res. 39, 316-322 (2011).

37. Livak, K. J. \& Schmittgen, T. D. Analysis of relative gene expression data using real-time quantitative PCR and the 2(-Delta Delta C(T)) Method. Methods 25, 402-408 (2011).

38. Li, J. et al. Combined proteomics and metabolism analysis unravels prominent roles of antioxidant system in the prevention of alfalfa (Medicago sativa L.) against salt stress. Int. J. Mol. Sci. 21, 909 (2020).

39. Phaladiganon, P., Kim, S. B., Chen, V. C. P. \& Jiang, W. Principal component analysis-based control charts for multivariate nonnormal distributions. Expert. Syst. Appl. 40, 3044-3054 (2013).

\section{Acknowledgements}

This work was supported by National Science and Technology Major Project (2018ZX08012001).

\section{Author contributions}

Conceptualization, F.L., S.Z.; Methodology, W.F., P.Z.; Investigation, W.F., P.Z., Y.Z., W.Z.; Writing, F.L., S.Z., M.Q.; Funding Acquisition, S.Z.; Resources, F.L, S.Z.; Supervision, S.Z.

\section{Competing interests}

The authors declare no competing interests.

\section{Additional information}

Supplementary Information The online version contains supplementary material available at https://doi. org/10.1038/s41598-021-81637-2.

Correspondence and requests for materials should be addressed to F.L. or S.Z.

Reprints and permissions information is available at www.nature.com/reprints.

Publisher's note Springer Nature remains neutral with regard to jurisdictional claims in published maps and institutional affiliations.

(c) (i) Open Access This article is licensed under a Creative Commons Attribution 4.0 International License, which permits use, sharing, adaptation, distribution and reproduction in any medium or format, as long as you give appropriate credit to the original author(s) and the source, provide a link to the Creative Commons licence, and indicate if changes were made. The images or other third party material in this article are included in the article's Creative Commons licence, unless indicated otherwise in a credit line to the material. If material is not included in the article's Creative Commons licence and your intended use is not permitted by statutory regulation or exceeds the permitted use, you will need to obtain permission directly from the copyright holder. To view a copy of this licence, visit http://creativecommons.org/licenses/by/4.0/.

(C) The Author(s) 2021 